\title{
Bisdemethoxycurcumin in combination with $\alpha$-PD-LI antibody boosts immune response against bladder cancer
}

This article was published in the following Dove Press journal:

OncoTargets and Therapy

22 May 2017

Number of times this article has been viewed

\author{
Yiqun Shao' \\ Wenjing Zhu \\ Jun $\mathrm{Da}^{\prime}$ \\ Mingxi $X u^{\prime}$ \\ Yiwei Wang' \\ Juan Zhou' \\ Zhong Wang'
}

'Department of Urology, Shanghai Ninth People's Hospital, Shanghai JiaoTong University School of Medicine, Shanghai, People's Republic of China; ${ }^{2}$ Department of Urology, Yueyang Hospital of integrated Traditional Chinese and Western Medicine, Shanghai University of Traditional Chinese Medicine, Shanghai, People's Republic of China

Correspondence: Zhong Wang Department of Urology, Shanghai Ninth People's Hospital, 639 Zhizaoju

Road, Shanghai 2000 II, People's

Republic of China

Tel +8621232716995116

Fax +862163136856

Email wangzhongshjy@gmail.com

\begin{abstract}
Curcumin was recently discovered to strengthen immune response through multiple mechanisms. Cytotoxic $\mathrm{CD} 8^{+} \mathrm{T}$-cells play a critical role in modulating anticancer immune response, but is severely restricted by T-cell exhaustion. Bladder carcinomas express PD-L1 and can abrogate $\mathrm{CD} 8^{+} \mathrm{T}$-cell response. Thus, we hypothesized that bisdemethoxycurcumin, a natural dimethoxy derivative of curcumin, may provide a favorable environment for T-cell response against bladder cancer when used in combination with $\alpha$-PD-L1 antibody. Immunocompetent C56BL/6 mouse models bearing subcutaneous or lung metastasized MB79 bladder cancer were established to validate this conjecture. We found that bisdemethoxycurcumin significantly increased intratumoral $\mathrm{CD}^{+} \mathrm{T}$-cell infiltration, elevated the level of IFN- $\gamma$ in the blood, and decreased the number of intratumoral myeloid-derived suppressor cells. Furthermore, $\alpha$-PD-L1 antibody protected these amplified CD8 ${ }^{+} \mathrm{T}$-cells from exhaustion, and therefore facilitated the secretion of IFN- $\gamma$, granzyme B, and perforin through these CD ${ }^{+} \mathrm{T}$-cells. As a result, this combination treatment strategy significantly prolonged survival of intraperitoneal metastasized bladder cancer bearing mice, suggesting that bisdemethoxycurcumin in combination with $\alpha$-PD-L1 antibody may be promising for bladder cancer patients.
\end{abstract}

Keywords: bladder cancer, immunotherapy, bisdemethoxycurcumin, PD-L1, combination therapy, metastasis

\section{Introduction}

Bladder cancer, the second most common genitourinary malignancy, is threatening human beings by its invasive and metastatic behavior. Conventional adjuvant chemotherapies such as combinations of platinum, gemcitabine, and ifosfamide usually fail to show survival advantages. Alternative nonsurgical therapeutic strategies are critical to improve patient survival. The immune system plays a critical role in controlling tumor growth, but malignancies, including bladder cancer, can escape immune attack through multiple pathways. ${ }^{1,2}$ Any method that can improve immune response against bladder cancer will provide opportunities for better outcome, especially for patients in whom first-line chemotherapy failed to control tumor progression. ${ }^{3}$

In immune cells, $\mathrm{CD}^{+} \mathrm{T}$-cell is one of the most effective cytotoxic cells in mediating tumor-specific attack. However, there are suffocating restraints in $\mathrm{CD} 8^{+}$ T-cell-based immunotherapy, such as immune checkpoint PD-1/PD-L1 axis, and the tumor immunosuppressive microenvironment including local Treg cells. Pathology studies have proven that most bladder cancer cells express PD-L1, ${ }^{4,5}$ which can profoundly impair the effects of cytotoxic CD8 ${ }^{+} \mathrm{T}$-cells (CTLs). In addition, tumors can develop an immunosuppressive microenvironment via different pathways, 
and consequently decrease the amount of tumor infiltrating lymphocytes (TILs). These two factors in combination, can result in suppressed CTL homing and weakened CTL function, and finally abrogate the spontaneous immune attack against bladder cancer.

Recently, antibodies for PD-1/PD-L1 pathway displayed favorable results in strengthening the activity of $\mathrm{CD} 8^{+} \mathrm{T}$-cells in clinical trials for bladder cancer. ${ }^{6,7}$ If there is a method to improve the immune response via immune checkpoint independent ways, it can improve the effects of checkpoint inhibitors. Concerning the immunosuppressive environment, intratumoral Treg cells and myeloid-derived suppressor cells (MDSCs) play critical roles. Curcumin, a widely studied component of turmeric, has been proven to facilitate immune response against different solid malignancies via multiple pathways. ${ }^{8-10}$ However, poor bioavailability has limited the application of curcumin. ${ }^{11}$ Thus, analogs of curcumin, such as bisdemethoxycurcumin (BDMC), may be promising drugs for clinical application. ${ }^{12}$ Given the fact that curcumin can mediate immune response, we conjectured that BDMC could promote immune attack against bladder cancer, and the effects could be strengthened when used in combination with PD-1/PD-L1 antibody.

In this study, we tried to validate our hypothesis that BDMC treatment and PD-1/PD-L1 blockade can promote spontaneous immune response against bladder cancer. We established immunocompetent C56BL/6 mouse models bearing subcutaneous (s.c.) or metastatic bladder cancer, and then treated them with BDMC and/or $\alpha-\mathrm{PD}-\mathrm{L} 1$ antibody. We discovered that low dose BDMC, in combination with $\alpha-\mathrm{PD}-\mathrm{L} 1$ antibody, displayed favorable effects in facilitating immune response and significantly prolonged mouse survival.

\section{Materials and methods Ethics approval}

The ethics committees from Shanghai Ninth People's Hospital and Shanghai Yueyang Hospital have approved this animal study, and the approval was obtained prior to the commencement of the study. All animal studies were performed following the guidelines and regulations of the animal care committees of Shanghai Ninth People's Hospital and Yueyang Hospital.

\section{Animals, cells, and chemicals}

Female C57BL/6 mice aged 7-8 weeks were purchased from Shanghai experimental animal center and maintained properly.

The MB49 mouse bladder carcinoma cells were maintained in Dulbecco's Modified Eagle's Medium with 10\% heat-inactivated fetal bovine serum, $2 \mathrm{mmol} / \mathrm{L} \mathrm{L-glutamine,}$
$1 \mathrm{mmol} / \mathrm{L}$ sodium pyruvate, and $100 \mathrm{U} / \mathrm{mL}$ penicillinstreptomycin.

BDMC (Sigma-Aldrich Co., St Louis, MO, USA) was dissolved in dimethyl sulfoxide at $20 \mathrm{mM}$ stock solution. Working concentrations were freshly prepared before use. Anti-mouse PD-L1 antibody was purchased from Bio X Cell (West Lebanon, NH, USA) and diluted in PBS.

\section{Tumor models}

To establish s.c. bladder cancer models, $1 \times 10^{6} \mathrm{MB} 49$ cells in $100 \mu \mathrm{L}$ PBS were injected in the right shaved flank. For lung metastasis models, $2 \times 10^{5}$ MB49 cells in $100 \mu \mathrm{L}$ PBS were administrated intravenously in the tail vein. One week post-tumor inoculation (day 7), the mice were randomized into different groups, each having at least ten mice per group as follows: vehicle control (PBS), BDMC single-drug treatment (BDMC), $\alpha$-PD-L1 antibody single-drug treatment ( $\alpha$-PD-L1), and combined treatment (combination). All treatments were then started. BDMC was administrated intravenously at $3 \mathrm{mg} / \mathrm{kg}$ body weight every 3 days for 2 weeks (metastasis models) or 4 weeks (s.c. models). $\alpha$-PD-L1 antibody was administrated intraperitoneally three times a week at $200 \mu \mathrm{g}$ for 2 weeks (metastasis models) or 4 weeks (s.c. models). Tumor volume was measured with caliper and calculated with the following formula: volume = $\left(\right.$ length $\times$ width $\left.^{2}\right) / 2$. Survival rate and mouse body weight were monitored.

\section{Sample processing}

For mice to be prepared for enzyme-linked immunosorbent assay (ELISA) and flow cytometry analysis, five mice from each group were anesthetized by intraperitoneal administration of ketamine ( $9 \mathrm{mg} / \mathrm{mL}$ in saline) and xylazine $(0.9 \mathrm{mg} / \mathrm{mL}$ in saline). Peripheral blood was collected from the tail when the mice were warmed up by heating lamp. Then the mice were sacrificed, and the spleens, s.c. tumor tissues, lungs, and TDLNs were harvested. The spleens were mashed, received red blood cell lysis, and passed through $40 \mu \mathrm{m}$ cell strainers. Also, lymph nodes were mashed and passed through strainers. Tumors and lungs were disaggregated by razors and incubated at $37^{\circ} \mathrm{C}$ for 1 hour in Roswell Park Memorial Institute 1640 medium with collagenase type IV $(2 \mathrm{mg} / \mathrm{mL}$, Sigma-Aldrich Co.), DNase $(0.1 \mathrm{mg} / \mathrm{mL}$, Sigma-Aldrich Co.), hyaluronidase (0.1 mg/mL, Sigma-Aldrich Co.), and bovine serum albumin ( $0.2 \mathrm{mg} / \mathrm{mL}$, Sigma-Aldrich Co.). Cell suspensions were passed through $100 \mu \mathrm{m}$ cell strainers to remove aggregates. All the cells mentioned previously were then washed with staining buffer (Biolegend, San Diego, CA, USA) and were ready for flow antibody incubation. 


\section{ELISA}

Serum IFN- $\gamma$ level was tested by ELISA (R\&D Systems, Inc., Minneapolis, MN, USA) following the manufacturer's instructions.

\section{Flow cytometry}

The following antibodies were purchased from Biolegend: anti-CD3 (clone 17A2), anti-CD45 (clone 30-F11), anti-CD4 (clone GK1.5), anti-CD8 $\alpha$ (clone 53-6.7), anti-IFN- $\gamma$ (clone XMG1.2), anti-CD11b (clone M1/70), and anti-Gr-1 (clone RB6-8C5). The following conjugated antibodies were obtained from eBioscience (San Diego, CA, USA): anti-granzyme B (clone NGZB) and anti-PD-1 (clone J43). Live cells were determined by 7AAD viability staining (Biolegend). For cell surface markers, cells were stained with antibodies at room temperature for 30 minutes. For intracellular antigen detection, an intracellular staining kit containing fixation/ permeabilization reagents from eBioscience was used. Flow cytometry analyses were performed on BD LSRFortessa X-20 (BD Biosciences, San Jose, CA, USA). s.c. tumor models

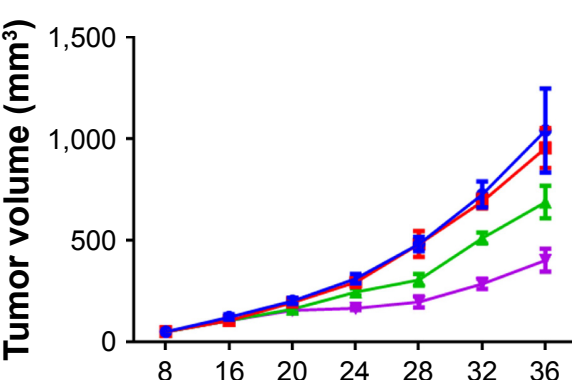

Days after tumor inoculation

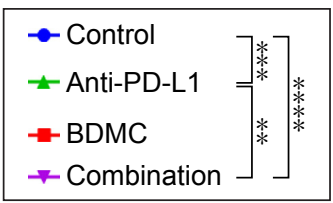

All gating strategies were determined by fluorescence minus one. Flowjo 10 was used to analyze the flow data.

\section{Statistical analysis}

Survival curves were illustrated using Kaplan-Meyer method. Statistical analysis was determined by one-way analysis of variance with Dunn's multiple comparison. All the analysis was performed on GraphPad Prism 5. All column data were presented as mean \pm standard error of the mean. $P<0.05$ was regarded as statistically significant.

\section{Results \\ Combination treatment controlled tumor growth and prolonged mouse survival}

We evaluated the volumes of s.c. tumors and monitored the survival of mice receiving different treatment regimens in both s.c. tumor and metastasis models. Low dose BDMC alone did not delay tumor growth (Figure 1A) in s.c. tumor models, but did have a trend in prolonging survival in both models, although the effect was modest (Figure 1B and C).

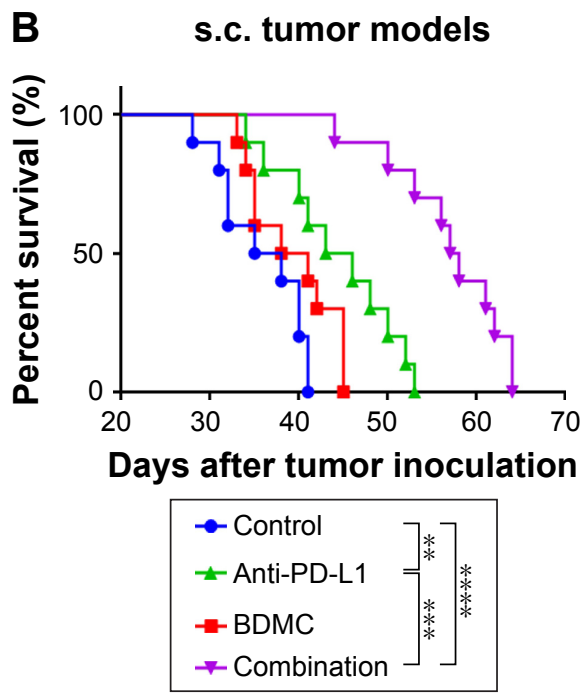

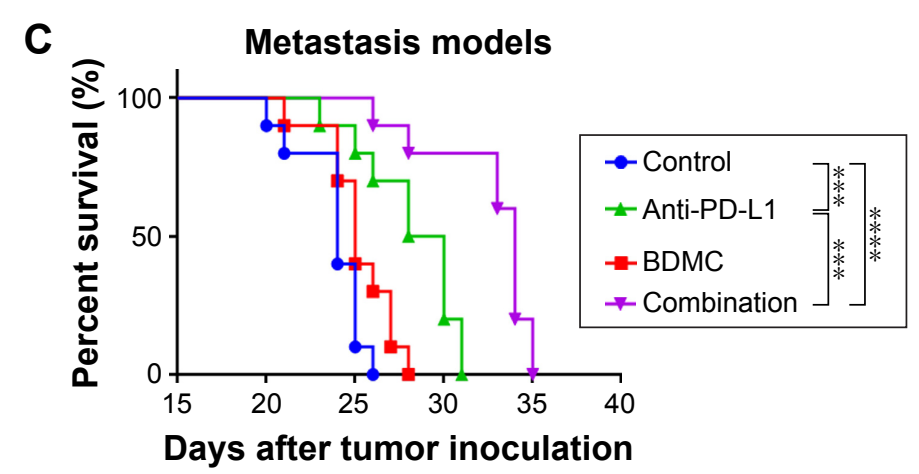

Figure I Combined treatment of BDMC and $\alpha$-PD-LI antibody inhibited tumor progression and significantly increased survival of mice bearing s.c. and metastatic bladder cancer.

Notes: (A) Tumor volumes were measured by caliper. (B) The survival curves of mice bearing s.c. bladder cancer were monitored. (C) The survival curves of mice with metastatic bladder cancer. $n=10$ group. $* * p<0.01$, $* * * P<0.001$, and $* * * * P<0.0001$. Data were presented as mean $\pm S E M$.

Abbreviations: BDMC, bisdemethoxycurcumin; s.c., subcutaneous; SEM, standard error of the mean. 
In addition, BDMC treatment did not reduce the body weight of mice (data not shown). $\alpha$-PD-L1 antibody alone, however, not only shrank the tumor, but also prolonged survival of mice. For mice receiving phosphate-buffered saline (PBS), the median survival of s.c. tumor models and lung metastasis models was 36.5 days and 24 days, respectively; while in $\alpha$-PD-L1 antibody treatment alone groups, the median survival was 44.5 days ( $P=0.0033$ ) and 29 days $(P=0.0007)$, respectively. Furthermore, combined treatment displayed much stronger benefit both in controlling tumor progression and prolonging survival. On day 36 , the median volume of tumors in the control group was $1,040 \mathrm{~mm}^{3}$, while it was $402 \mathrm{~mm}^{3}$ in the combination treatment group $(P<0.0001)$, ie, combined therapy significantly delayed tumor growth. The median survival in combination group in s.c. tumor models and metastasis models was 57.5 days $(P<0.0001)$ and 34 days $(P<0.0001)$, respectively. The combination strategy of BDMC and $\alpha$-PD-L1 antibody illustrated much more powerful antitumor effects than single drug treatment.

\section{BDMC increased the number and activity of $\mathrm{CD} 8^{+} \mathrm{T}$-cells}

To reveal the effects of BDMC on lymphocytes, we analyzed the phenotypes of splenic lymphocytes and cells in tumor-draining lymph nodes (TDLNs) from different groups in both models. In splenocytes, BDMC significantly increased the proportion of $\mathrm{CD}^{+} \mathrm{T}$-cells (Figure $2 \mathrm{~A}-\mathrm{D}$ ) in both models. This elevated number of $\mathrm{CD}^{+}$cells indicated that BDMC helped in $\mathrm{CD}^{+} \mathrm{T}$-cell survival. In TDLNs from s.c. tumor models, again, an increased number of $\mathrm{CD} 8^{+} \mathrm{T}$-cells were discovered in BDMC treated mice, while PD-L1 antibody had no effect on the number of T-cells (Figure 3A and B). These elevated $\mathrm{CD} 8^{+} \mathrm{T}$-cells (by BDMC) expressed a higher level of IFN- $\gamma$ (Figure 3C and D) and granzyme B (Figure 3E and $\mathrm{F}$ ) than that in control or $\alpha$-PD-L1 antibody alone groups, indicating that BDMC not only elevated the number of $\mathrm{CD}^{+}$ T-cells in the TDLNs, but also strengthened their antitumor activity. Furthermore, serum IFN- $\gamma$ level was measured. Unsurprisingly, BDMC elevated serum IFN- $\gamma$ level in both s.c. and lung metastasis models (Figure $3 \mathrm{G}$ and $\mathrm{H}$ ), demonstrating that BDMC significantly strengthened immune response.

\section{$C D 8^{+} T$ cells were more infiltrated but restricted to exhaustion after BDMC treatment}

In both models, flow cytometry results showed a significantly larger number of $\mathrm{CD}^{+}$TILs in BDMC treated mice when compared with PBS control mice. BDMC alone resulted in a four-fold (s.c. model) (Figure 4A and B) or three-fold (lung metastasis model) (Figure 5A and B) increase of intratumoral

\section{A Control}

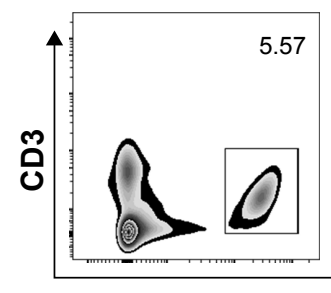

BDMC

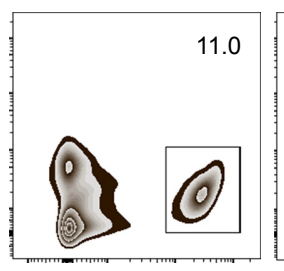

$\mathrm{CD}^{+}$

C

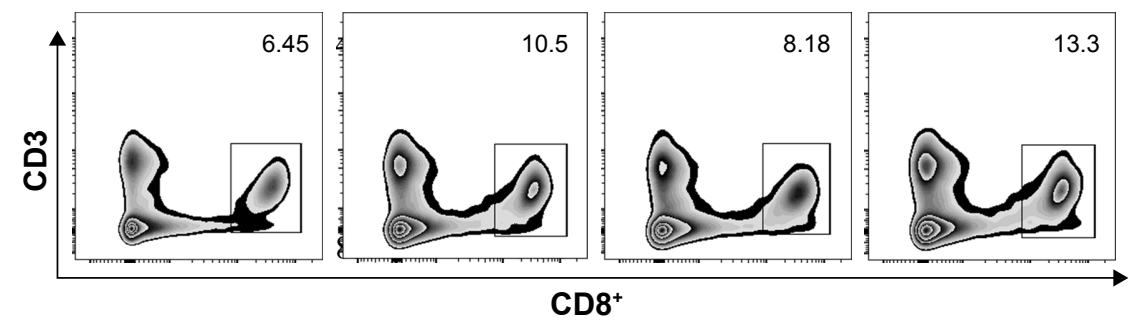

$\alpha-P D-L 1 \quad$ Combination

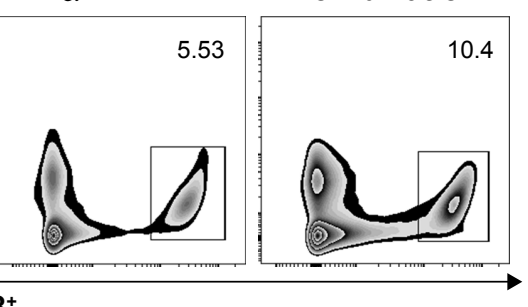



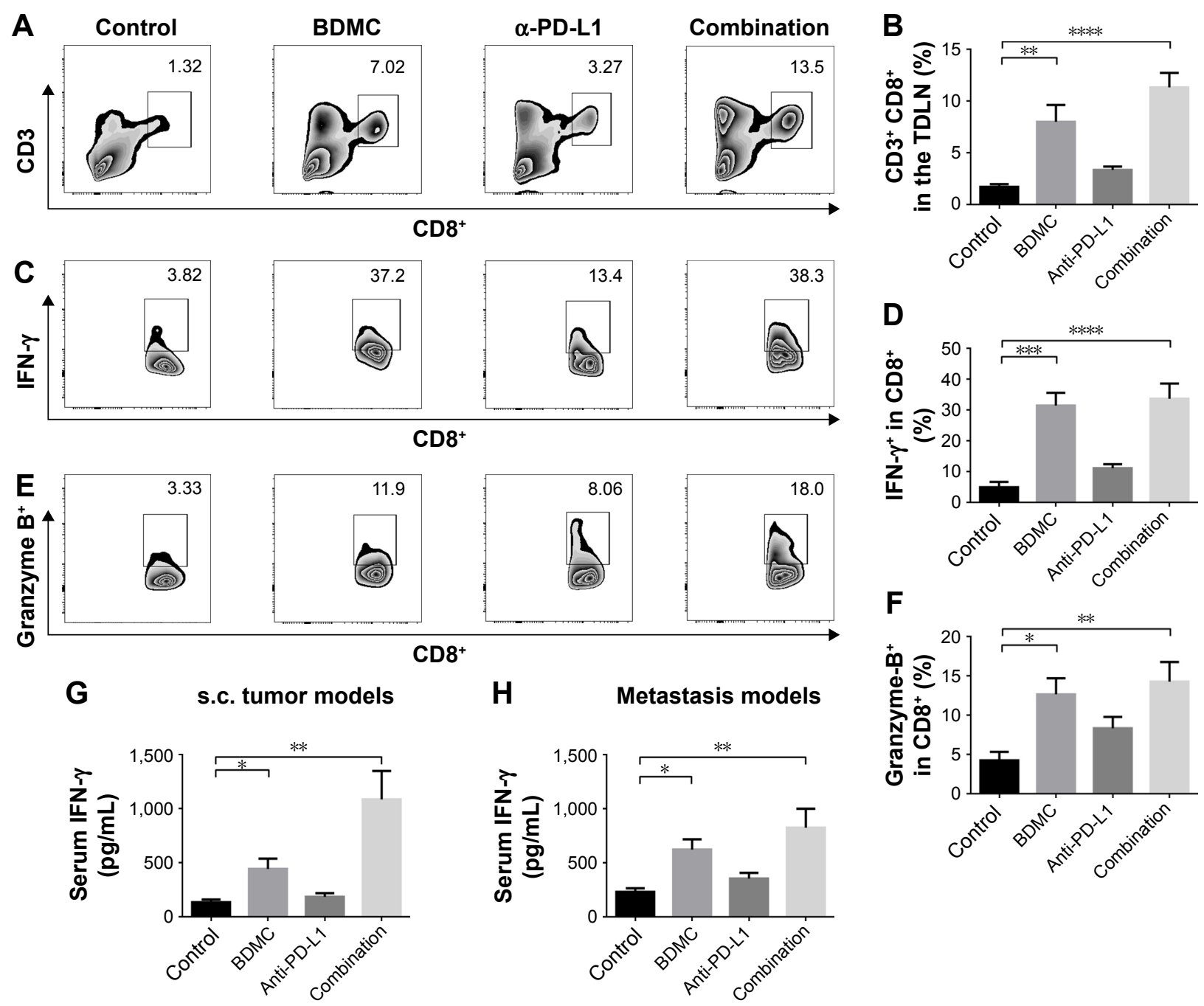

Figure 3 Combined treatment significantly strengthened CD8 $8^{+}$-cells in TDLNs.

Notes: Representative data (A) and pooled data (B) of proportions of CD8 ${ }^{+}$T-cells in TDLNs in different groups. Representative data of proportions of IFN- $\gamma^{+}(\mathbf{C})$ and granzyme $\mathrm{B}^{+}(\mathbf{E})$ cells in $\mathrm{CD} 8^{+}$cells from TDLNs. Pooled data of frequencies of IFN- $\gamma^{+}(\mathbf{D})$ and granzyme $\mathrm{B}^{+}(\mathbf{F})$ cells in CD8 $8^{+}$cells in TDLNs from different groups. ELISA assay also revealed that BDMC treatment significantly elevated serum IFN- $\gamma$ level in s.c. tumor models $(\mathbf{G})$ and metastasis models $(\mathbf{H}) . n=5 /$ group. $* P<0.05,{ }^{* * P}<0.0$ I, $* * * P<0.00$ I, and $* * * * P<0.0001$. Data were presented as mean $\pm \mathrm{SEM}$.

Abbreviations: TDLNs, tumor-draining lymph nodes; ELISA, enzyme-linked immunosorbent assay; BDMC, bisdemethoxycurcumin; s.c., subcutaneous; SEM, standard error of the mean.

CD8 ${ }^{+}$T-cells. However, in both models, most of these intratumoral $\mathrm{CD} 8^{+} \mathrm{T}$-cells were $\mathrm{PD}-1$ positive (Figure $4 \mathrm{C}$ and $\mathrm{D}$; Figure $5 \mathrm{C}$ and $\mathrm{D}$ ), while very few of them expressed IFN- $\gamma$ (Figure 4E and F; Figure 5E and F). This status was also seen in PBS control mice, which indicated that although more $\mathrm{CD}^{+}{ }^{+}$CTLs infiltrated the tumor after low dose BDMC treatment, these cells failed to present their antitumor ability. The exhausted status of intratumoral $\mathrm{CD} 8^{+} \mathrm{T}$-cells may account for the limited anticancer effect of BDMC treatment alone.

\section{$\alpha-P D-L I$ antibody boosted T-cell response}

We validated the role $\alpha-\mathrm{PD}-\mathrm{L} 1$ antibody played in both s.c. and lung metastasis models. Although $\alpha$-PD-L1 antibody alone did not display a statistically significant benefit for
CD8 ${ }^{+} \mathrm{T}$-cells in the spleen, it did have favorable effects in CTLs in TDLNs. $\alpha$-PD-L1 antibody elevated the secretion of IFN- $\gamma$ (Figure 3C and D) and granzyme B (Figure 3E and $\mathrm{F}$ ) by $\mathrm{CD}^{+} \mathrm{T}$-cells. In tumor tissues from both models, although there was no statistically significant elevation in the number of tumor infiltrated CD $8^{+}$T-cells, $\alpha-P D-L 1$ antibody down-regulated the expression of PD- 1 on intratumoral $\mathrm{CD} 8^{+}$ T-cells (Figure 4C and D; Figure 5C and D) and elevated the level of IFN- $\gamma$ in these cells (Figure 4E and F; Figure 5E and F), indicating that $\alpha$-PD-L1 antibody alone protected the function of intratumoral effector T-cells.

In mice receiving combined treatment of BDMC and $\alpha$-PD-L1 antibody, the immune response was markedly triggered. As mentioned above, BDMC had positive effects 
A

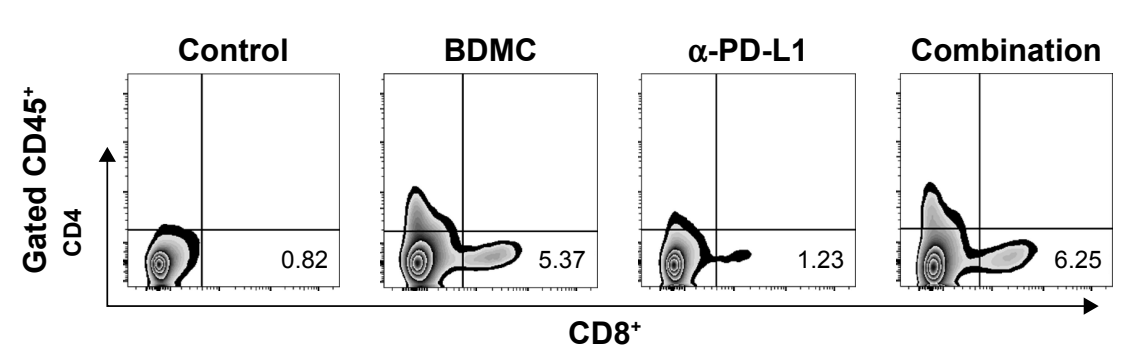

C

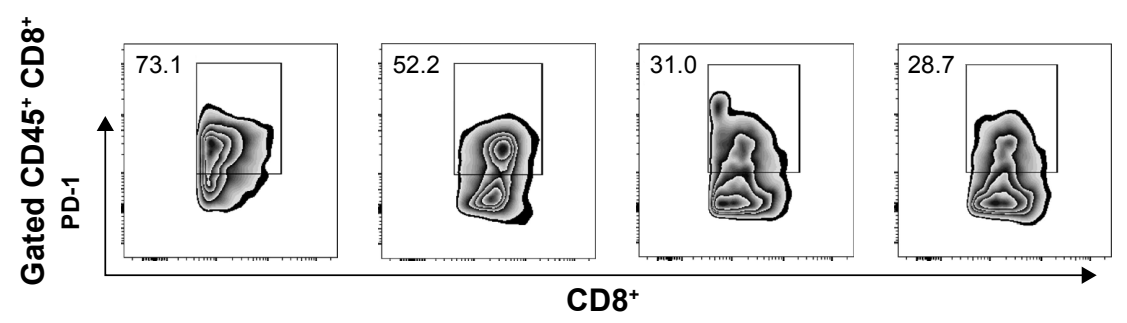

E

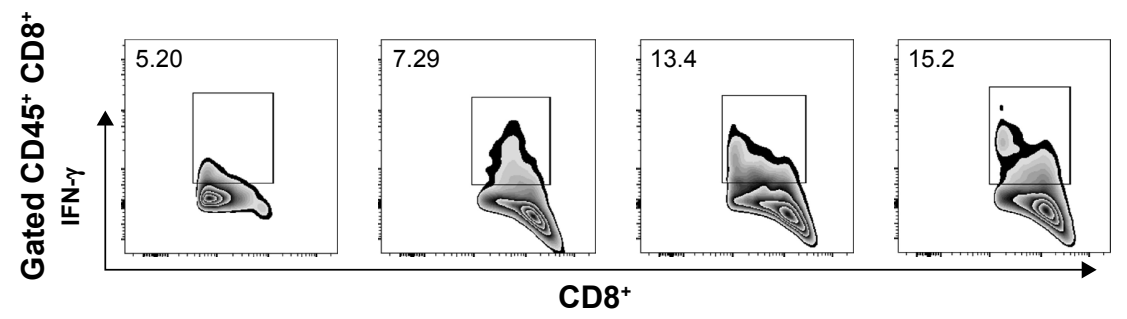

B

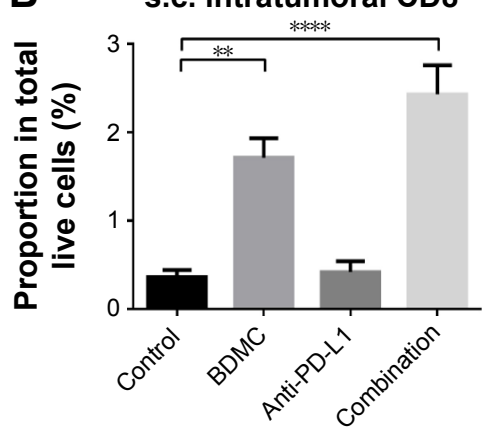

D s.c. intratumoral

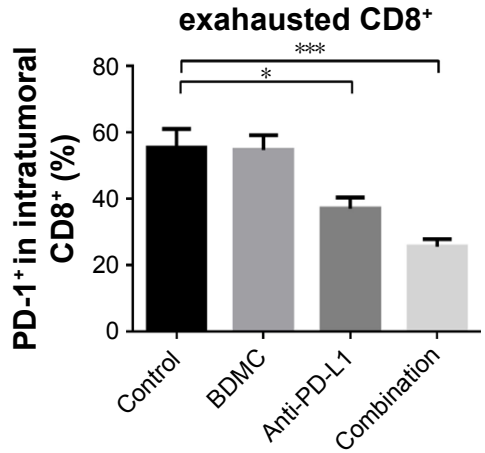

$\mathbf{F}$

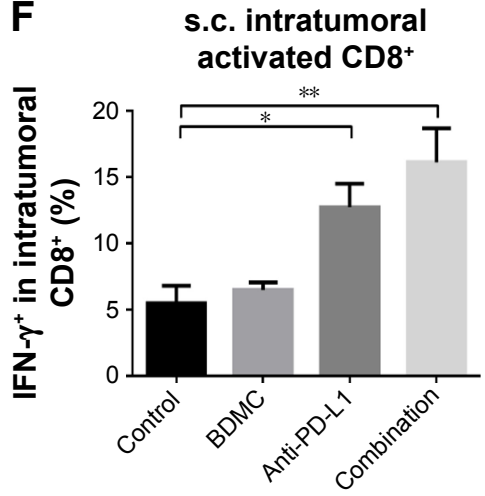

Figure 4 Combination treatment elevated the number and activity of intratumoral $\mathrm{CD}^{+} \mathrm{T}$-cells in s.c. tumors.

Notes: Representative data $(\mathbf{A})$ and pooled data $(\mathbf{B})$ of proportions of $C D 8^{+} \mathrm{T}$-cells in tumors from different groups. Representative data of proportions of PD- $I^{+}(\mathbf{C})$ and IFN- $\gamma^{+}(\mathbf{E})$ cells in intratumoral $C D 8^{+}$cells. Pooled data of frequencies of PD- $I^{+}(\mathbf{D})$ and IFN- $\gamma^{+}(\mathbf{F})$ cells in intratumoral $C D 8^{+}$cells from different groups. $n=5 / g r o u p . ~ * P<0.05$, $* * P<0.01$, $* * * P<0.001$, and $* * * * P<0.0001$. Data were presented as mean $\pm \mathrm{SEM}$.

Abbreviations: s.c., subcutaneous; SEM, standard error of the mean.

on $\mathrm{CD} 8^{+} \mathrm{T}$-cell and activation, and $\alpha$-PD-L1 antibody protected the intratumoral $\mathrm{CD} 8^{+} \mathrm{T}$-cells from exhaustion. In the combination group, the number of $\mathrm{CD} 8^{+} \mathrm{T}$-cells in TDLNs from s.c. models was significantly improved (Figure $3 \mathrm{~A}$ and $\mathrm{B}$ ), along with improved functioning of $\mathrm{CD} 8^{+} \mathrm{T}$-cells (Figure 3C-F). In addition, a profoundly elevated secretion of IFN- $\gamma$ by intratumoral $\mathrm{CD} 8^{+} \mathrm{T}$-cells was discovered, which was much higher than that in BDMC or $\alpha$-PD-L1 antibody treatment alone group (Figure 4E and F; Figure 5E and F). Furthermore, the proportion of exhausted T-cells in the combination group was much lower than in BDMC alone group (Figure 4C and D; Figure 5C and D).
Combination treatment increased the number of intratumoral CTLs, facilitated immune response, and protected effector T-cells from exhaustion.

\section{Combination treatment reduced the proportion of MDSCs in s.c. models}

MDSCs are highly immune suppressive in the tumor microenvironment. We analyzed the intratumoral MDSCs in both models. BDMC treatment alone had a trend in controlling the number of MDSCs, but $\alpha$-PD-L1 antibody treatment and combined treatment significantly decreased the number of MDSCs in the tumor (Figure 6A and B) in s.c. models. 
A

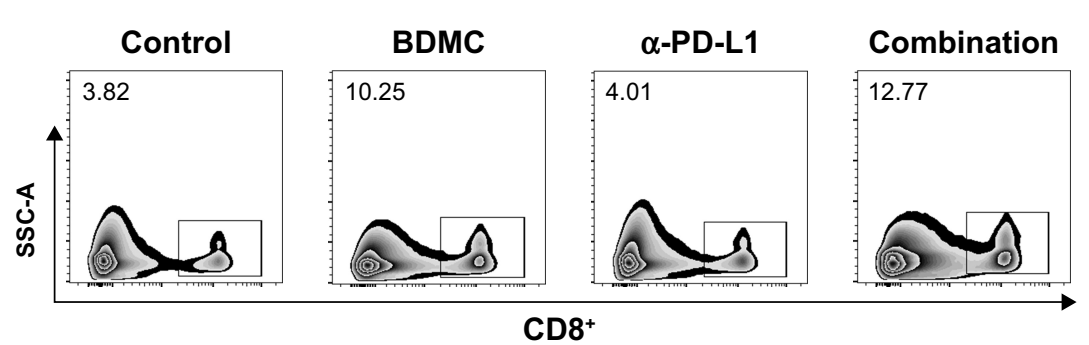

C

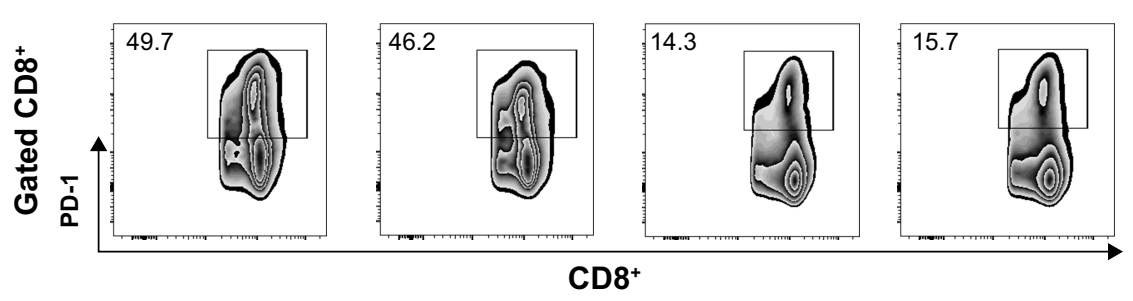

$\mathbf{E}$

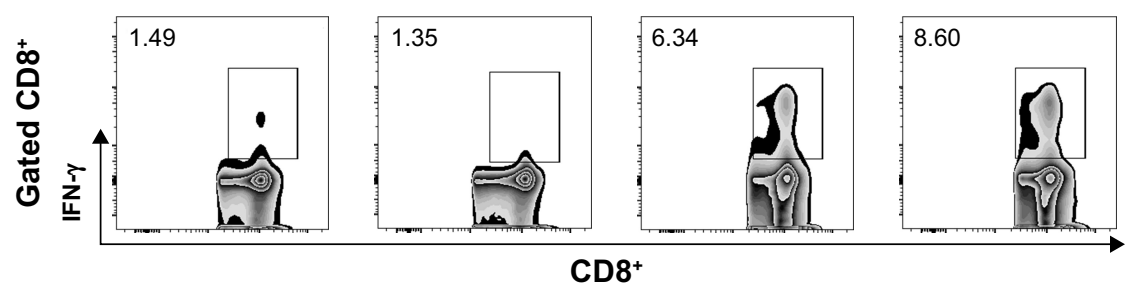

B Pulmonary intratumoral

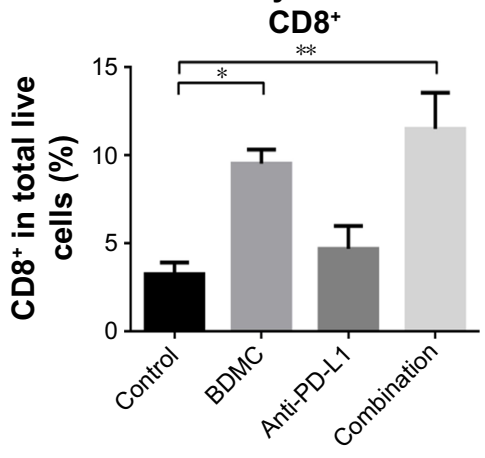

D Pulmonary exhausted

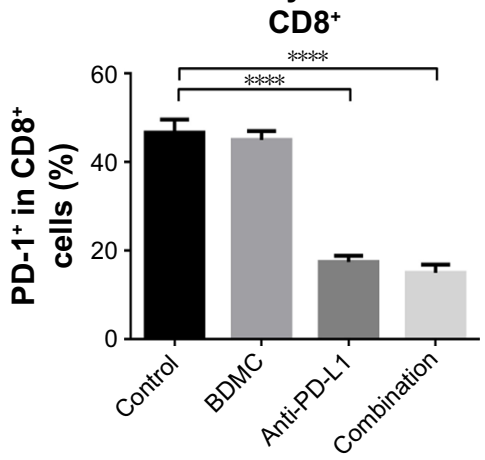

F Pulmonary activated

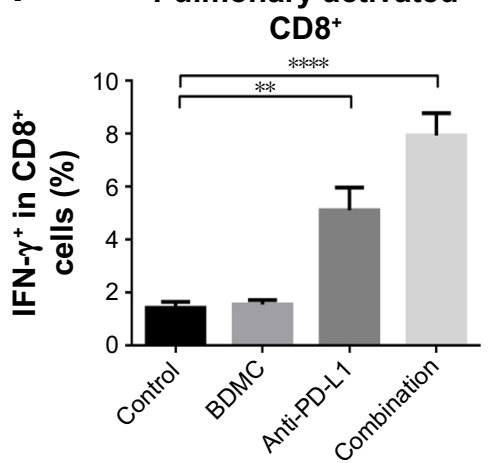

Figure 5 Combination treatment boosted immune response in pulmonary metastasized bladder cancer.

Notes: Representative data (A) and pooled data (B) of proportions of CD8 ${ }^{+}$T-cells in tumors from different groups. Representative data of proportions of PD- ${ }^{+}(\mathbf{C})$ and IFN- $\gamma^{+}(\mathbf{E})$ cells in pulmonary intratumoral $C D 8^{+}$cells. Pooled data of frequencies of PD- $I^{+}(\mathbf{D})$ and IFN- $\gamma^{+}(\mathbf{F})$ cells in intratumoral CD8 $8^{+}$cells from different groups. $\mathrm{n}=5$ /group. $* \mathrm{P}<0.05, * * \mathrm{P}<0.01$, and $* * * * \mathrm{P}<0.000$ I. Data were presented as mean $\pm \mathrm{SEM}$.

Abbreviation: SEM, standard error of the mean.

In lung metastasis models, however, comparatively fewer MDSCs were discovered in the lung. No statistically significant difference was seen among groups (data not shown).

\section{Discussion}

$\mathrm{CD} 8^{+} \mathrm{T}$-cells play critical roles in immune attack against tumors. However, the underlying mechanisms affecting T-cell activation, differentiation, function, and survival are still largely unknown. Any method which can facilitate T-cell activity will undoubtedly help in cancer immunotherapy.
Curcumin, and its analogs, were recently validated as a potential immune stimulator. ${ }^{8,10}$ However, the underlying mechanisms by which curcumin modulates immune response are still elusive. It has been demonstrated that curcumin could facilitate immune response by preventing $\mathrm{T}$-cell apoptosis and altering the tumor microenvironment. ${ }^{13-15}$ Besides, the clinical application of curcumin has been restricted because of its poor bioavailability. ${ }^{11}$ In addition, malignancies, especially solid tumors, can weaken immune attack by cytotoxic T-cells through multiple pathways. PD-1/PD-L1 pathway 
A

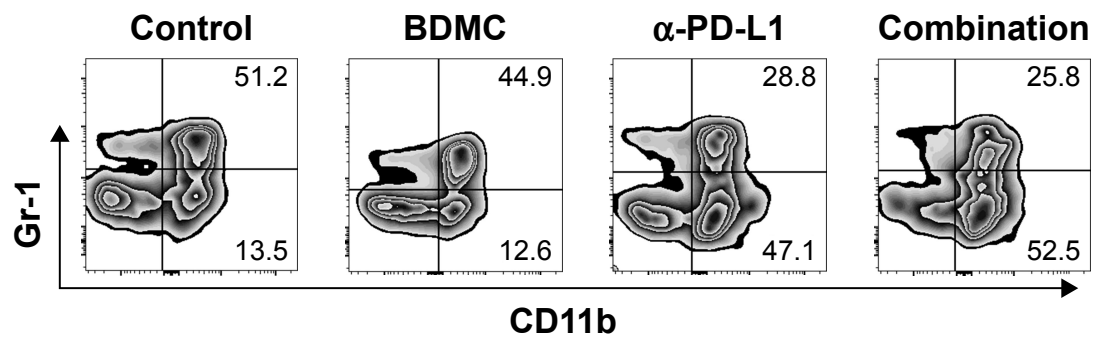

B

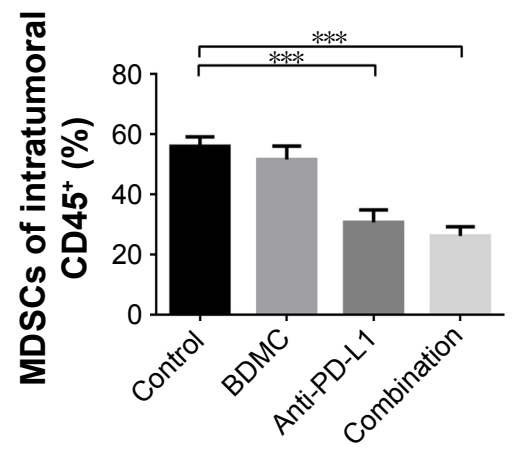

Figure 6 Combination treatment inhibited MDSC proliferation in s.c. tumor models.

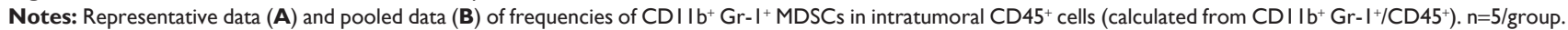
$* * * P<0.001$. Data were presented as mean \pm SEM.

Abbreviations: MDSC, myeloid-derived suppressor cell; s.c., subcutaneous; SEM, standard error of the mean.

plays one of the most important roles in inhibiting immune response. ${ }^{16,17}$ Without controlling the immune checkpoint modulators, T-cell-mediated antitumor immune response will be severely impaired.

In the current study, we evaluated the combination treatment of BDMC, an analog of curcumin which has elevated bioavailability and stability, and $\alpha$-PD-L1 antibody for bladder cancer in immunocompetent mouse models. We demonstrated that mice receiving low-dose BDMC treatment had strengthened T-cell response. Elevated levels of IFN- $\gamma$ and granzyme B secretion were monitored. However, although experiments on peripheral lymphocytes and the number of intratumoral T-cells revealed favorable results, single-agent treatment of BDMC still had modest effects in prolonging mouse survival and intratumoral T-cell activity. In tumor tissues, most of the infiltrated T-cells displayed an exhausted phenotype, which may be correlated with the expression of PD-L1 on bladder cancer cells. On the other hand, the single-agent administration of $\alpha-P D-L 1$ antibody maintained the activity of $\mathrm{CD} 8^{+} \mathrm{T}$-cells, while the number of infiltrated T-cells was still far from satisfactory. Thus, the combination treatment of both BDMC and $\alpha$-PD-L1 antibody successfully boosted immune response against bladder cancer, and significantly prolonged survival. CTLs, regardless of whether in the TDLNs or in the tumors, secreted significantly higher levels of IFN- $\gamma$ and granzyme B, and consequently controlled tumor progression. Besides, potent immune suppressor cells, MDSCs, were also inhibited after BDMC treatment. We observed decreased expression of PD-1 on intratumoral CD8 ${ }^{+}$ T-cells, which was not seen in single-agent treated mice. We conjectured that it might be associated with the decreased number of intratumoral MDSCs. ${ }^{18}$

Clinical trials for bladder cancer using different kinds of immunotherapies such as adoptive cell transfer and
PD-1/PD-L1 antibodies are ongoing. However, solid tumors escape immune response through different mechanisms, including immune suppressive local environment and immune checkpoint ligands/receptors. Any method which can facilitate immune response will be valuable for cancer immunotherapy. BDMC can be used in combination with other therapies, and theoretically reach at least additive, or even synergistic effects. The authors are looking forward to the future, in which cocktail immunotherapy, combing adoptive cell transfer, adjuvants such as BDMC, and checkpoint inhibitors, can achieve the most powerful anticancer effects. Of note, the potential side effects of BDMC and related combination treatment are not negligible. As an analog of curcumin, BDMC shares most of the side effects of curcumin, including allergies, gastrointestinal problems such as nausea and diarrhea, etc. ${ }^{11,12}$ However, these side effects are usually mild and subside fast. ${ }^{12}$

In conclusion, this is the first study demonstrating that BDMC treatment in combination with $\alpha$-PD-L1 antibody can suppress bladder cancer progression in vivo and prolong mouse survival. Combination treatment boosted immune response by stimulating $\mathrm{CD} 8^{+} \mathrm{T}$-cell activity and suppressing MDSCs. Our results suggest that the combination of BDMC and $\alpha$-PD-L1 antibody may be a promising therapeutic regimen for treatment of bladder cancer patients.

\section{Disclosure}

The authors report no conflicts of interest in this work.

\section{References}

1. Vanneman M, Dranoff G. Combining immunotherapy and targeted therapies in cancer treatment. Nat Rev Cancer. 2012;12(4):237-251.

2. Kamat AM, Hahn NM, Efstathiou JA, et al. Bladder cancer. Lancet. 2016;388(10061):2796-2810.

3. Donin NM, Lenis AT, Holden S, et al. Immunotherapy for the treatment of urothelial carcinoma. J Urol. 2017;197(1):14-22. 
4. Wang X, Teng F, Kong L, Yu J. PD-L1 expression in human cancers and its association with clinical outcomes. Onco Targets Ther. 2016;9: 5023-5039.

5. Inman BA, Sebo TJ, Frigola X, et al. PD-L1 (B7-H1) expression by urothelial carcinoma of the bladder and BCG-induced granulomata: associations with localized stage progression. Cancer. 2007;109(8): 1499-1505.

6. Powles T, Eder JP, Fine GD, et al. MPDL3280A (anti-PD-L1) treatment leads to clinical activity in metastatic bladder cancer. Nature. 2014; 515(7528):558-562.

7. Rosenberg JE, Hoffman-Censits J, Powles T, et al. Atezolizumab in patients with locally advanced and metastatic urothelial carcinoma who have progressed following treatment with platinum-based chemotherapy: a single-arm, multicentre, phase 2 trial. Lancet. 2016;387(10031): 1909-1920.

8. Lu Y, Miao L, Wang Y, et al. Curcumin micelles remodel tumor microenvironment and enhance vaccine activity in an advanced melanoma model. Mol Ther. 2016;24(2):364-374.

9. Zhou J, Donatelli SS, Gilvary DL, et al. Therapeutic targeting of myeloid-derived suppressor cells involves a novel mechanism mediated by clusterin. Sci Rep. 2016;6:29521.

10. Bill MA, Bakan C, Benson DJ Jr, Fuchs J, Young G, Lesinski GB. Curcumin induces proapoptotic effects against human melanoma cells and modulates the cellular response to immunotherapeutic cytokines Mol Cancer Ther. 2009;8(9):2726-2735.

11. Liu H, Xu H, Jiang Y, et al. Preparation, characterization, in vivo pharmacokinetics, and biodistribution of polymeric micellar dimethoxycurcumin for tumor targeting. Int J Nanomedicine. 2015;10:6395-6410.
12. Basile V, Ferrari E, Lazzari S, Belluti S, Pignedoli F, Imbriano C. Curcumin derivatives: molecular basis of their anti-cancer activity. Biochem Pharmacol. 2009;78(10):1305-1315.

13. Bhattacharyya S, Mandal D, Saha B, Sen GS, Das T, Sa G. Curcumin prevents tumor-induced T cell apoptosis through Stat-5a-mediated Bcl-2 induction. J Biol Chem. 2007;282(22):15954-15964.

14. Bao B, Ahmad A, Li Y, et al. Targeting CSCs within the tumor microenvironment for cancer therapy: a potential role of mesenchymal stem cells. Expert Opin Ther Targets. 2012;16(10):1041-1054.

15. Jiang GM, Xie WY, Wang HS, et al. Curcumin combined with FAPalphac vaccine elicits effective antitumor response by targeting indolamine-2,3-dioxygenase and inhibiting EMT induced by TNF-alpha in melanoma. Oncotarget. 2015;6(28):25932-25942.

16. Zou W, Wolchok JD, Chen L. PD-L1 (B7-H1) and PD-1 pathway blockade for cancer therapy: Mechanisms, response biomarkers, and combinations. Sci Transl Med. 2016;8(328):324r-328r.

17. Li B, Zhu X, Sun L, et al. Induction of a specific CD8+ T-cell response to cancer/testis antigens by demethylating pre-treatment against osteosarcoma. Oncotarget. 2014;5(21):10791-10802.

18. Dufait I, Schwarze JK, Liechtenstein T, et al. Ex vivo generation of myeloid-derived suppressor cells that model the tumor immunosuppressive environment in colorectal cancer. Oncotarget. 2015;6(14): 12369-12382.
OncoTargets and Therapy

\section{Publish your work in this journal}

OncoTargets and Therapy is an international, peer-reviewed, open access journal focusing on the pathological basis of all cancers, potential targets for therapy and treatment protocols employed to improve the management of cancer patients. The journal also focuses on the impact of management programs and new therapeutic agents and protocols on

\section{Dovepress}

patient perspectives such as quality of life, adherence and satisfaction. The manuscript management system is completely online and includes a very quick and fair peer-review system, which is all easy to use. Visit http://www.dovepress.com/testimonials.php to read real quotes from published authors. 